\title{
Electronic Excitations and Luminescence of SrMgF4 Single Crystals
}

Article in Physics of the Solid State · March 2014

DOI: $10.1134 / S 106378341403024 X$

CITATION

1

8 authors, including:

\section{V.A. Pustovarov}

Ural Federal University

300 PUBLICATIONS 1,025 CITATIONS

SEE PROFILE

\section{Sergey I. Omelkov}

University of Tartu

35 PUBLICATIONS 94 CITATIONS

SEE PROFILE
READS

51
Igor N. Ogorodnikov

Ural Federal University

197 PUBLICATIONS 848 CITATIONS

SEE PROFILE

\section{I. Isaenko}

Sobolev Institute of Geology and Mineralogy 278 PUBLICATIONS 2,417 CITATIONS

SEE PROFILE

Some of the authors of this publication are also working on these related projects: 


\title{
Electronic Excitations and Luminescence of $\mathrm{SrMgF}_{4}$ Single Crystals
}

\author{
V. A. Pustovarov, ${ }^{1}$ I. N. Ogorodnikov, ${ }^{1,}{ }^{*} \quad$ S. I. Omelkov, ${ }^{2}$ L. I. Isaenko, ${ }^{3}$ \\ A. P. Yelisseyev, ${ }^{3}$ A. A. Goloshumova, ${ }^{3}$ S. I. Lobanov, ${ }^{3}$ and P. G. Krinitsyn ${ }^{3}$ \\ ${ }^{1}$ Ural Federal University named after the first President of Russia B.N. Yeltsin, ul. Mira 19, Yekaterinburg, 620002 Russia \\ ${ }^{2}$ Institute of Physics, University of Tartu, Estonia \\ ${ }^{3}$ Институт геологии и минералогии СО РАН, 630058 Новосибирск, Россия
}

(Received June 13, 2013)

\begin{abstract}
The electronic and crystal structures of $\mathrm{SrMgF}_{4}$ single crystals grown by the Bridgman method have been investigated. The undoped $\mathrm{SrMgF}_{4}$ single crystals have been studied using low-temperature $(T=$ $10 \mathrm{~K}$ ) time-resolved fluorescence optical and vacuum ultraviolet spectroscopy under selective excitation by synchrotron radiation $(3.7-36.0 \mathrm{eV})$. Based on the measured reflectivity spectra and calculated spectra of the optical constants, the following parameters of the electronic structure have been determined for the first time: the minimum energy of interband transitions $E_{\mathrm{g}}=12.55 \mathrm{eV}$, the position of the first exciton peak $E_{n=1}=11.37 \mathrm{eV}$, the position of the maximum of the 'exciton' luminescence excitation band at $10.7 \mathrm{eV}$, and the position of the fundamental absorption edge at $10.3 \mathrm{eV}$. It has been found that photoluminescence excitation occurs predominantly in the region of the low-energy fundamental absorption edge of the crystal and that, at energies above $E_{\mathrm{g}}$, the energy transfer from the matrix to luminescence centers is inefficient. The exciton migration is the main excitation channel of photoluminescence bands at 2.6-3.3 and 3.3 $-4.2 \mathrm{eV}$. The direct photoexcitation is characteristic of photoluminescence from defects at $1.8-2.6$ and $4.2-5.5 \mathrm{eV}$.
\end{abstract}

DOI: $10.1134 / \mathrm{S} 106378341403024 \mathrm{X}$

\section{INTRODUCTION}

More than 30 years ago, crystalline magnesiumstrontium tetrafluoride $\mathrm{SrMgF}_{4}$ (SMF) belonging to the $\mathrm{SrF}_{2}-\mathrm{MgF}_{2}$ system was synthesized and investigated for the first time [1, 2]. Structural investigations of SMF were performed at room temperature by Ishizawa et al. [3]. The SMF crystals are described in the monoclinic system with symmetry space group $P 112_{1}(\mathrm{Amam})$ and the lattice parameters $a=$ 782.49(8), $b=749.30(7), c=1692.48(17) \mathrm{pm}, \gamma=$ 105.041(11) .

The disordering of the SMF crystal lattice is responsible for a number of important properties of this compound. First, from the theory it follows that $\mathrm{SrMgF}_{4}$ crystals can exhibit ferroelectric properties with the Curie temperature $T_{\mathrm{C}}=420 \mathrm{~K}$ [4]. Second, the presence of a large number of nonequivalent positions for the introduction of impurity atoms in the structure makes it possible to control the luminescent properties of the SMF compound over a wide spectral range. This aspect of the problem has been investigated in a number of works on the photoluminescence (PL) of SMF crystals doped with rare-earth elements: the PL emission spectra of $\mathrm{Ce}^{3+}$ impurity ions in non equivalent positions of the SMF crystal lattice were examined under excitation in the wavelength range from 100 to $600 \mathrm{~nm}$ at temperatures of $17-300 \mathrm{~K}$ [5, 6]; the $\mathrm{Sm}^{2+} \mathrm{PL}$ emission spectra were studied at an excitation energy of $2.54 \mathrm{eV}(488 \mathrm{~nm})$ in host lattices of SMF [7] and $\mathrm{Ba}_{1-\delta} \mathrm{Sr}_{\delta} \mathrm{MgF}_{4}(\delta<0.55)$ [8]; and

\footnotetext{
* igor.ogorodnikov@bk.ru
}

the PL emission spectra of europium and terbium impurity ions in different charge states were investigated in $[9,10]$. Third, the disordering of the crystal lattice should affect the spectral and luminescent properties of intrinsic lattice defects in SMF. We are aware of only one research work [11] devoted to the consideration of this aspect of the problem. It was found that nano-crystalline SMF samples prepared from aqueous solutions with the subsequent annealing exhibit an intense broadband luminescence with long afterglow. This luminescence is attributed to defects, because the related material $\mathrm{BaMgF}_{4}$ synthesized by a similar method remains optically transparent in the wavelength range from 180 to $8000 \mathrm{~nm}$ (up to $6.9 \mathrm{eV}$ ). The position of the maximum of the emission band depends on the temperature and duration of annealing. This suggests that the material contains intrinsic lattice defects responsible for the observed PL emission [11].

From general considerations, it is clear that the disordering of the crystal lattice should have a decisive influence on the specific features of the relaxation of electronic excitations in the $\mathrm{SrMgF}_{4}$ host lattice. However, we are unaware of the studies carried out in this direction. Therefore, the purpose of the present work was to perform an experimental study of the electronic structure, relaxation, and radiative decay of low-energy electronic excitations in undoped $\mathrm{SrMgF}_{4}$ single crystals. This study was carried out using low-temperature time-resolved fluorescence optical and vacuum ultraviolet spectroscopy under selective excitation by synchrotron radiation over a wide energy range. 


\section{DETAILS OF THE EXPERIMENT}

The $\mathrm{SrMgF}_{4}$ compound was synthesized from $\mathrm{SrF}_{2}$ and $\mathrm{MgF}_{2}$ powders (special-purity grade). These powders, taken in a stoichiometric ratio, were placed in a glassy carbon crucible located in a quartz ampoule. Powders with a highly developed surface always adsorb a lot of moisture from the atmosphere. In order to remove it, the mixture of powders was heated in a dynamic vacuum of $10^{-1} \mathrm{~Pa}$ for $24 \mathrm{~h}$ at a temperature of $500^{\circ} \mathrm{C}$, after which the ampoule was hermitically sealed. The synthesis of $\mathrm{SrMgF}_{4}$ was performed in a single-zone furnace heated to the melting temperature of the binary components for $1 \mathrm{~h}$ with occasional stirring.

The $\mathrm{SrMgF}_{4}$ single crystals were grown by the Bridgman method in a two-zone furnace. The charge was placed in a glassy carbon crucible with a conical bottom located in a quartz ampoule evacuated to a residual pressure of $10^{-1} \mathrm{~Pa}$ and hermetically sealed in the presence of the fluorine agent $\mathrm{CF}_{4}$. The ampoule was moved from the hot zone of the furnace heated to $1200^{\circ} \mathrm{C}$ into the cold zone $\left(700^{\circ} \mathrm{C}\right)$ at a speed of $1 \mathrm{~mm}$ per day, so that the temperature gradient in the growth zone was equal to $10-20^{\circ} \mathrm{C} / \mathrm{cm}$. At the end of the experiment, the crystal was rapidly cooled in the turned-off furnace mode. The size of transparent blocks was $10 \times 10 \times 5 \mathrm{~mm}$.

Samples of the $\mathrm{SrMgF}_{4}$ single crystal had the form of optically transparent plates $(7 \times 7 \times 1.5 \mathrm{~mm}$ in size), which were cut perpendicular to the (010) plane. The sample surface was polished to laser quality. Based on preliminary spectroscopic measurements, two samples (SMF\#1 and SMF\#2) with different luminescent properties were chosen for further detailed investigation. In all cases, when the sample number is not specified, this means that there are no noticeable differences in the specific properties of the two samples. In other cases, the sample number is clearly specified. The orientation of the crystallographic axes of the $\mathrm{SrMgF}_{4}$ samples was arbitrary with respect to the polarization vector $\mathbf{E}$ of the synchrotron radiation. The only exception was the large (010) plane of sample SMF\#1, whose normal was always perpendicular to the polarization vector $\mathbf{E}$ of the synchrotron radiation.

The structural investigation was carried out using the pure and optically transparent single crystals $\left(0.19 \times 0.13 \times 0.11 \mathrm{~mm}^{3}\right.$ in size $)$ on a Bruker APEX DUO automated diffractometer $(\mathrm{MoK} \alpha$ radiation, $\lambda=71.073 \mathrm{pm}$, graphite monochromator, CCD detector). All the calculations were performed with the SHELXTL program packages (Bruker AXS Inc. (2004), APEX (Version 1.08), SAINT (Version 7.03), SADABS (Version 2.11), and SHELXTL (Version 6.12), Bruker Advanced X-Ray Solutions. Madison, Wisconsin, United States). The graphical visualization of the structure was carried out with the BS program (T.C. Ozawa and S.J. Kang, "Balls \& Sticks:

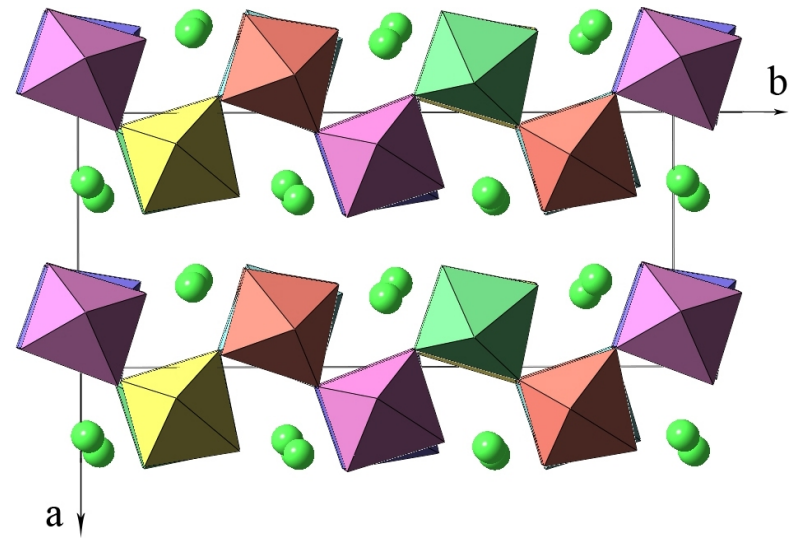

Figure 1. Crystallographic structure of $\mathrm{SrMgF}_{4}$. Circles are strontium atoms, and polyhedra are $\mathrm{MgF}_{6}$ groups.

Easy-to-use structure visualization and animation creating program", Version 1.42 (released 2002-05-25), J. Appl. Cryst. 37, 679 (2004)). The results of the structural investigation are presented in Table 1.

It was found that, in the $\mathrm{SrMgF}_{4}$ structure, there are six nonequivalent positions of strontium atoms, six nonequivalent positions of magnesium atoms, and twenty four nonequivalent positions of fluorine atoms. In general, the structure can be described as a layered one. The normal to the plane of layers is oriented perpendicular to the $c$ axis. The layers are formed by chains that are oriented along the $b$ axis and consist of irregular vertex-shared $\mathrm{MgF}_{6}$ octahedra rotated with respect to each other (Fig. 1). The strontium atoms are located between the layers and, depending on the position, are surrounded by seven $(\mathrm{Sr} 1, \mathrm{Sr} 2)$, nine $(\mathrm{Sr} 3, \mathrm{Sr} 5)$, or ten $(\mathrm{Sr} 4, \mathrm{Sr} 6)$ fluorine atoms (the coordination numbers were determined by means of the construction of Dirichlet polyhedra with the XShell program [13]). The obtained results are in good agreement with the previously published data [3]. It should be noted that the structure was solved by different methods. We used the standard setting $P 12_{1} 1\left(\beta=105.030^{\circ}\right)$, whereas in [3], the structure was described as $P 112_{1}\left(\gamma=105.041^{\circ}\right)$.

The PL spectra in the spectral range from 1.2 to $6.2 \mathrm{eV}$, the PL excitation spectra, and the reflectivity spectra at an angle of $17^{\circ}(3.7-36.0 \mathrm{eV})$ were measured under selective photoexcitation by synchrotron radiation at the SUPERLUMI experimental station [14] (Hamburg Synchrotron Radiation Laboratory (HASYLAB) at the German Electron Synchrotron DESY, Hamburg, Germany). Synchrotron radiation pulses from the DORIS storage ring had a Gaussian shape (full width at half-maximum FWHM= $130 \mathrm{ps}$ ) with a repetition period of $96 \mathrm{~ns}$. The measurements were performed in the steady-state (time-integrated (TI)) mode and in the time-resolved mode at temperatures 
Table 1. Crystallographic data for $\mathrm{SrMgF}_{4}$ and conditions of diffraction experiments

\begin{tabular}{|c|c|}
\hline Parameter & Description \\
\hline Crystal system & Monoclinic \\
\hline Space group & $P 2_{1}$ \\
\hline \multirow[t]{3}{*}{ Unit cell parameters, pm } & $a=747.36(5)$ \\
\hline & $b=1688.35(12)$ \\
\hline & $c=780.10(5)$ \\
\hline Angle $\beta$, deg & $105.030(2)$ \\
\hline Unit cell volume, $\mathrm{pm}^{3}$ & $9.5066(11) \times 10^{-6}$ \\
\hline Number of formula units per unit cell $Z$ & 12 \\
\hline Density (calculated), $\mathrm{g} / \mathrm{cm}^{3}$ & 3.939 \\
\hline Molecular weight & 187.93 \\
\hline Wavelength, pm & 71.073 \\
\hline$\mu(\operatorname{MoK} \alpha), \mathrm{mm}^{-1}$ & 17.130 \\
\hline Structure amplitude $F(000)$ & 1032 \\
\hline Scan range $\Theta,^{\circ}$ & $2.41-28.28$ \\
\hline \multirow[t]{3}{*}{ Range of Miller indices $h, k, l$} & $-9 \leq h \leq 9$ \\
\hline & $-22 \leq k \leq 22$ \\
\hline & $-10 \leq l \leq 10$ \\
\hline Number of measured reflections & 11895 \\
\hline Number of unique reflections & $4694\left[R_{\mathrm{int}}=0.0322\right]$ \\
\hline Completeness of data collection on $\Theta=28.28 \%$ & 99.9 \\
\hline \multirow[t]{2}{*}{ Absorption correction method } & Semiempirical method with inclusion \\
\hline & of the intensities of equivalent reflections \\
\hline Maximum and minimum transmittances & 0.2615 и 0.1410 \\
\hline Refinement method & Full-matrix least-squares method for $\mathrm{F}^{2}$ \\
\hline Number of reflections/constraints/parameters & $4694 / 13 / 325$ \\
\hline$S$-фактор по $\mathrm{F}^{2}$ & 0.750 \\
\hline$R$-фактор $[I>2 \sigma(I)]$ & $R 1=0.0235, w R 2=0.0560$ \\
\hline$R$-фактор (все данные) & $R 1=0.0321, w R 2=0.0604$ \\
\hline Absolute structure parameter & $0.008(5)$ \\
\hline Maximum and minimum residual electron densities, e/ $\AA^{3}$ & 0.765 and -0.730 \\
\hline
\end{tabular}

The description of the parameters corresponds to [12]: $F=f\left(h, k, l, x_{\mathrm{c}}, y_{\mathrm{c}}, z_{\mathrm{c}}\right)$ is the structural factor, where $x_{\mathrm{c}}, y_{\mathrm{c}}, z_{\mathrm{c}}$ are the crystallographic coordinates (below in the formulas: $F_{0}$ is the experimentally obtained value, and $F_{\mathrm{c}}$ is the calculated value); $R_{\mathrm{int}}=\sum\left|F_{0}^{2}-\left\langle F_{\mathrm{c}}^{2}\right\rangle\right| / \sum F_{0}^{2}$, where $\left\langle F_{\mathrm{c}}^{2}\right\rangle$ is the average of all the measured equivalents; $\mu(\mathrm{MoK} \alpha)$ is the absorption coefficient of $\operatorname{MoK} \alpha \mathrm{X}$-ray radiation; $I$ is the intensity of the peaks; $\sigma(I)$ is the variance of the intensity; $R$-factor (residual factor) characterizes the accuracy of the performed investigation, three variants of it were used: $S=\left[\sum\left|w\left(F_{0}^{2}-F_{\mathrm{c}}^{2}\right)^{2}\right| /\left(N_{r}-N_{p}\right)\right]^{1 / 2}$, where $N_{r}$ is the number of unique reflections, $N_{p}$ is the number of refined parameters, $w$ is the weighting factor; $R 1=\sum|| F_{0}|-| F_{\mathrm{c}}|| / \sum\left|F_{0}\right| ; w R 2=\left[\sum w\left(F_{0}^{2}-F_{\mathrm{c}}^{2}\right)^{2} / \sum w F_{0}^{2}\right]^{1 / 2}$.

of 10 and $293 \mathrm{~K}$ with a continuous-flow liquid helium cryostat, which provided a vacuum of no worse than $5 \times 10^{-8} \mathrm{~Pa}$. The selective PL excitation by synchrotron radiation was carried out using a 2 -m vacuum monochromator equipped with Al- or Pt-coated interchangeable gratings (the spectral resolution was $0.32 \mathrm{~nm}$ ). The PL excitation spectra were normalized to equal numbers of photons incident on the sample. The PL spectra in the range from 1.2 to $6.2 \mathrm{eV}$ were measured using a 0.3-m ARC Spectra Pro-308i monochromator and a Hamamatsu R6358P photomultiplier. The time-resolved spectra were recorded in two independent time windows (TWs) synchronized with respect to the beginning of the excitation pulse: 0.8-18.1 ns (TW1) and 126.8-189.4 ns (TW2). The time-window parameters were chosen based on the PL decay kinetics.

\section{EXPERIMENTAL RESULTS}

The reflectivity spectra measured for the SMF crystal at $T=10$ and $293 \mathrm{~K}$ in the fundamental absorption edge region are shown in Fig. 2. Figure 3 presents the 


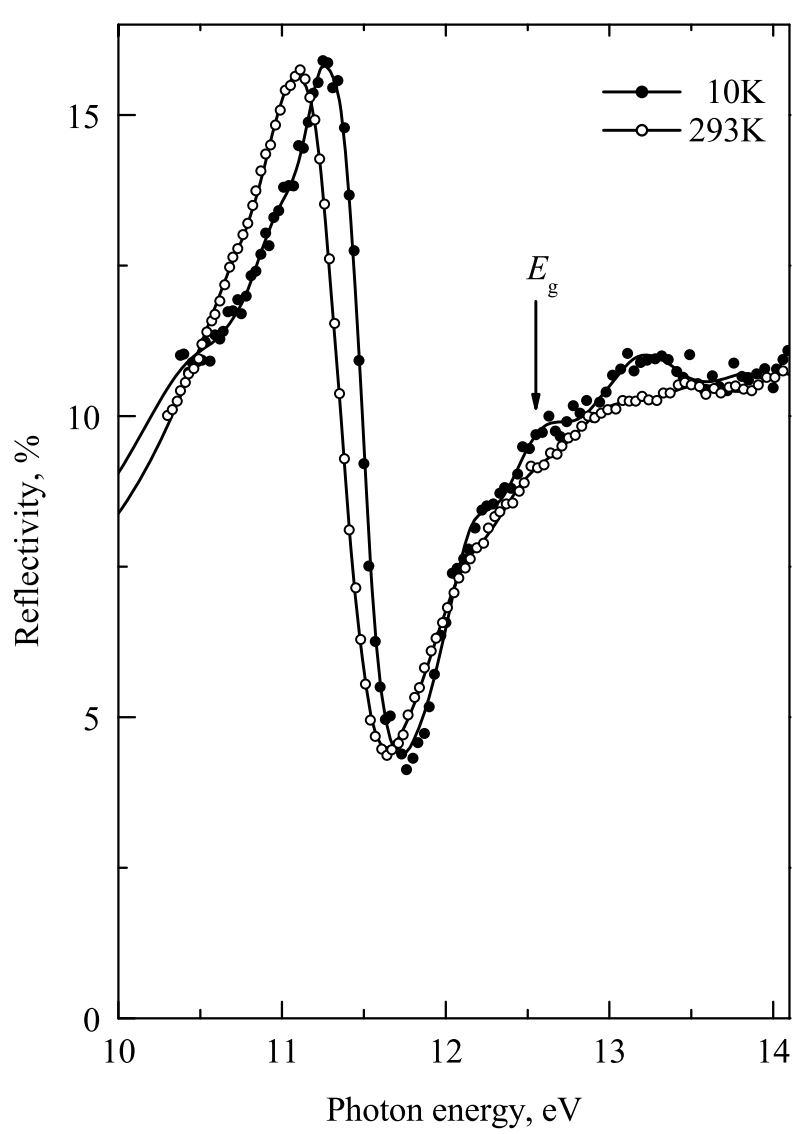

Figure 2. Reflectivity spectra of the $\mathrm{SrMgF}_{4}$ crystal at $T=10$ and $293 \mathrm{~K}$. Points are the experimental data, and solid lines show the result of the approximation. The vertical arrow indicates the band gap $E_{\mathrm{g}}$ at $10 \mathrm{~K}$.

experimental reflectivity spectrum measured for the SMF crystal in the energy range from 8 to $36 \mathrm{eV}$ at $293 \mathrm{~K}$ and the spectra of the optical constants calculated from this spectrum using the difference KramersKrönig method [15]. The reflectivity spectrum was initially measured in relative units. For the conversion to absolute values of the reflectivity, we used the normalization to the refractive index in the transparency region of the crystal (see Subsection 3.1).

At a low temperature $(T=10 \mathrm{~K})$, the $\mathrm{SrMgF}_{4}$ crystals exhibit an intense luminescence in a wide spectral range from 1.8 to $6.0 \mathrm{eV}$. The profile of the PL spectrum depends on the excitation photon energy, which indicates a superposition of several PL bands forming the observed luminescence spectrum. The PL spectra measured upon excitation by photons with different energies in the range from 9.5 to $25.8 \mathrm{eV}$ are shown in Figs. 4 and 5. In the PL spectra, there are four regions with partially overlapping spectra, namely, 1.8-2.6(I), 2.6-3.3 (II), 3.3-4.2 (III), and 4.2-5.5 eV (IV), which correspond to different PL bands. For brevity, these regions will be designated as PL-I, PL-II, etc.

For the excitation energy $E_{\text {ex }}$ ranging from 25.8 to

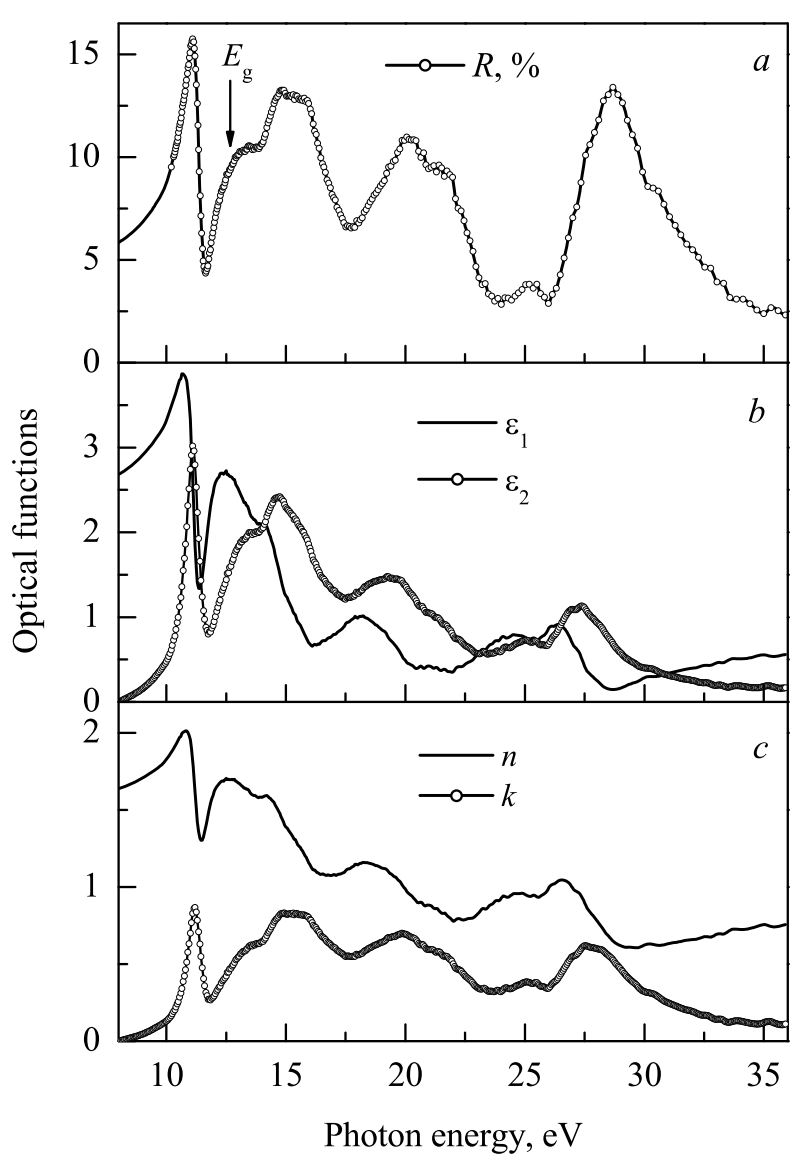

Figure 3. Spectra of the $\mathrm{SrMgF}_{4}$ crystal at $T=293$ $\mathrm{K}:(a)$ experimental reflectivity spectrum and $(b, c)$ spectra of the optical constants calculated using the Kramers-Krönig method.

$18.3 \mathrm{eV}$, the difference PL spectrum is observed in regions II and III (Fig. 4). When the excitation energy $E_{\text {ex }}$ changes from 11.2 to $10.8 \mathrm{eV}$, the PL intensity smoothly increases in the entire spectrum and the observed maximum of the PL spectrum in all cases is located in region III. The specific position of the observed maximum depends on the excitation energy. In the vicinity of this maximum, the luminescence intensity increases disproportionately. For the excitation energy ranging from 9.5 to $10.4 \mathrm{eV}$, the PL maximum is located at $3.75 \mathrm{eV}$, whereas in the excitation energy range of $10.6-25.8 \mathrm{eV}$, the PL maximum is observed at $3.65 \mathrm{eV}$ (Figs. 4 and 5). As the excitation energy increases in the intermediate range from 10.4 to $10.6 \mathrm{eV}$, the observed PL maximum gradually shifts from 3.75 to $3.65 \mathrm{eV}$, while the PL intensity smoothly decreases in region II at 3.0-3.1 eV and simultaneously increases in region IV at 5.0-5.1 eV. For $E_{\text {ex }}<10.3 \mathrm{eV}$, sample SMF\#1 does not exhibit luminescence in regions II-IV, whereas sample SMF\#2 is characterized by the PL intensity, which gradually decreases in the entire spectrum with a decrease in the excitation energy $E_{\text {ex }}$ 


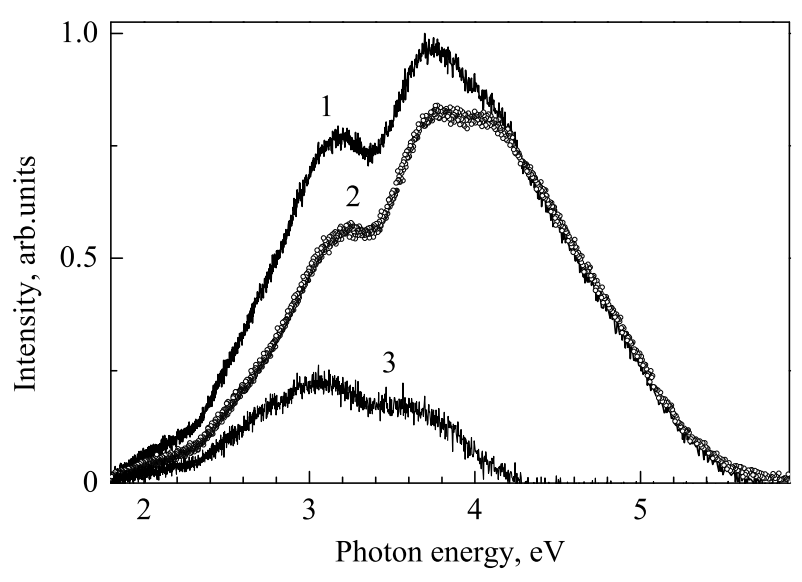

Figure 4. PL spectra of the $\mathrm{SrMgF}_{4}$ crystal measured at the temperature $T=10 \mathrm{~K}$ and excitation energies $E_{\text {ex }}=$ (1) 25.8 and (2) $18.3 \mathrm{eV}$. Curve 3 represents the difference between spectra 1 and 2 .

from 10.0 to $9.5 \mathrm{eV}$.

Figure 6 shows the PL excitation spectra measured during the monitoring of the luminescence at the energy Em in regions II-IV. All the spectra are characterized by an identical structure that consists of a broad band at $12.0 \mathrm{eV}$ and four excitation bands in the range from 10 to $11 \mathrm{eV}$, which are designated by the letters A $(10.2 \mathrm{eV}), \mathrm{B}(10.5 \mathrm{eV}), \mathrm{C}(10.7 \mathrm{eV})$, and D $(11.0 \mathrm{eV})$. Most of the differences in the PL excitation spectra (Fig. 6) are caused by changes in the intensities of these bands: the A band is observed only for sample SMF\#2, the B band is observed equally for both samples and corresponds to the PL excitation in region IV, the $\mathrm{C}$ band dominates in the PL excitation spectra of both samples, and the D band appears as a shoulder in the spectra of both samples.

The PL-I spectra measured at temperatures of 10 and $293 \mathrm{~K}$ for different excitation energies are shown in Fig. 7. At $293 \mathrm{~K}$, the PL spectrum in the range from 1.5 to $6.0 \mathrm{eV}$ consists of only one broad complex band at $2.1 \mathrm{eV}$. The low-energy slope of this PL band is located near the low-energy limit of the recording system, where a detailed analysis of the shape of the luminescence band is complicated. In this regard, Fig. 7 shows only three most intense elementary bands obtained by decomposition of the spectrum. At $10 \mathrm{~K}$, the PL band at $2.1 \mathrm{eV}$ has a low intensity (Fig. 4) and appears on the low-energy 'tail' of more intense low-temperature PL bands whose maxima are located in the visible and ultraviolet regions of the spectrum (Fig. 7).

More than $95 \%$ of the light sum is concentrated in microsecond and millisecond components of the PL decay kinetics, which are presented in our measurements in the form of a constant level-pedestal. Therefore, in most cases, the recorded time-resolved and time-integrated spectra have an identical profile;

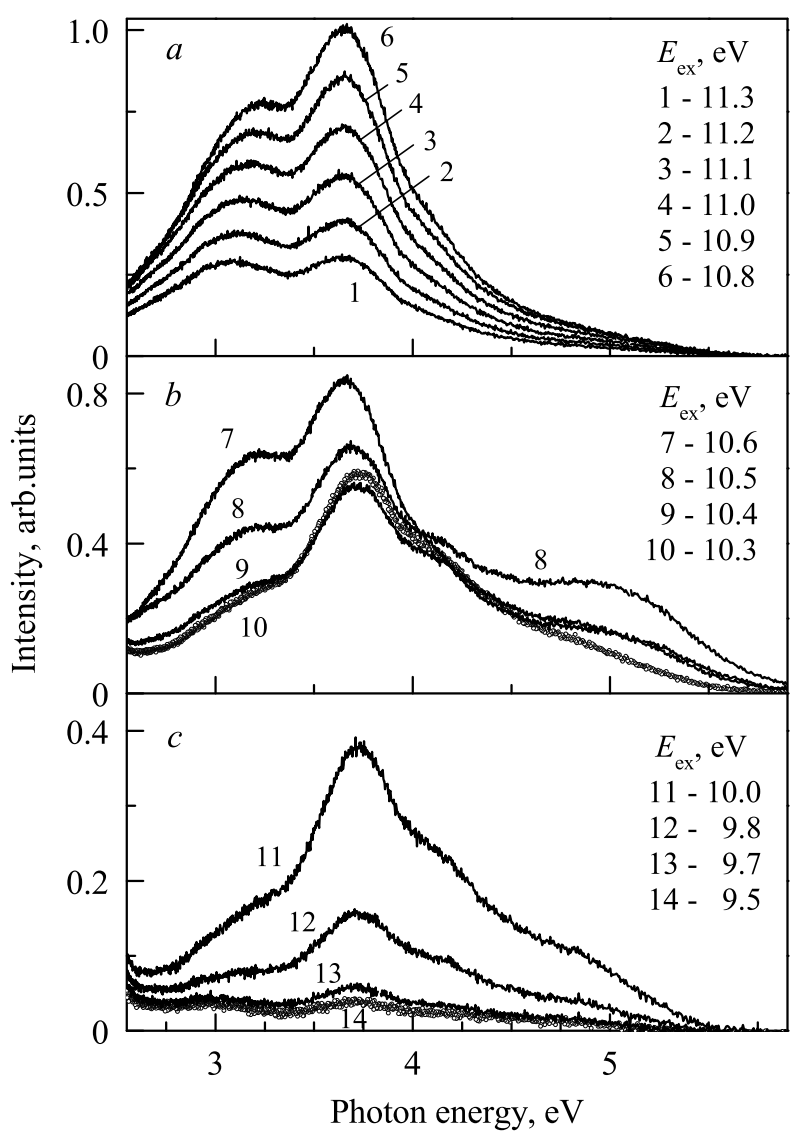

Figure 5. PL spectra of the $\mathrm{SrMgF}_{4}$ crystal measured at the temperature $T=10 \mathrm{~K}$ and different excitation energies $E_{\mathrm{ex}}$ for samples $(a, b) \mathrm{SMF} \# 1, \mathrm{SMF} \# 2$; and (c) SMF\#2.

hence, only the time-integrated spectra are presented in Figs. 2-7. In some cases, it is possible to separate a fast non-single-exponential component of the PL decay kinetics (Fig. 8). The fast component is excited at $10.5 \mathrm{eV}$ in a narrow energy range of approximately $0.15 \mathrm{eV}$. This component was formally approximated by the sum of two exponential components with decay time constants of 1.45 and $7.00 \mathrm{~ns}$ for the initial intensity ratio 21 . The numerical processing of the time-resolved spectra recorded in the time windows TW1 and TW2 allowed us to explicitly distinguish the spectrum of the fast PL component with a maximum at $4.6 \mathrm{eV}(\mathrm{FWHM}=1.15 \mathrm{eV})($ Fig. 8).

The PL-I excitation spectrum (Fig. 9) differs significantly from the excitation spectra of other PL bands. At $293 \mathrm{~K}$, the excitation spectrum is dominated by a narrow band at 9.9-10.0 eV and two broad bands at $8-9$ and $5.5-6.5 \mathrm{eV}$. In the range from 10.3 to $11.7 \mathrm{eV}$, the efficiency of the PL-I excitation is extremely low. With a further increase in the excitation energy, the PL-I intensity gradually increases to approximately $30 \%$ of the maximum in the region of $9.9-10.0 \mathrm{eV}$. At $T=10 \mathrm{~K}$, the PL-I excitation spectrum is characterized 


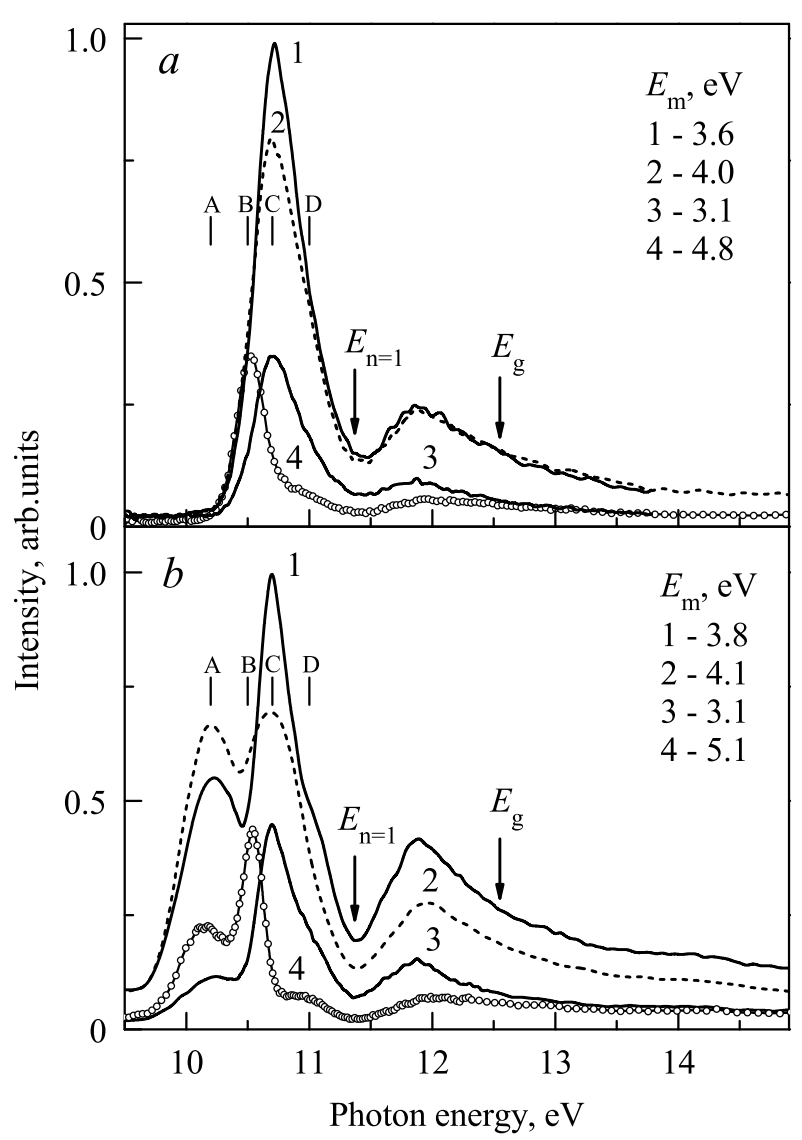

Figure 6. PL excitation spectra of the $\mathrm{SrMgF}_{4}$ crystals measured at the temperature $T=10 \mathrm{~K}$ and different values of $E_{\mathrm{m}}$ for samples (a) SMF\#1 and (b) SMF\#2. Vertical arrows indicate the position of the first exciton peak $\mathrm{E} E_{n=1}$ and the band gap $E_{\mathrm{g}}$.

Letters A-D designate the selected PL excitation bands.

by a doublet of the $\mathrm{B}$ and $\mathrm{D}$ bands, with the higher intensity of the B band.

\section{DISCUSSION}

\subsection{Spectra of the Optical Constants}

The reflectivity spectra were analyzed using two different methods: the oscillator model for analyzing the fundamental absorption edge in the energy range of $10-14 \mathrm{eV}$ at temperatures of 10 and $293 \mathrm{~K}$ (Fig. 2) and the Kramers-Krönig transform for the analysis in the energy range of $8-36 \mathrm{eV}$ at $293 \mathrm{~K}$.

Within the framework of the oscillator model [16, 17], the contribution of each oscillator $j$ to the complex dielectric constant of the optical material is given

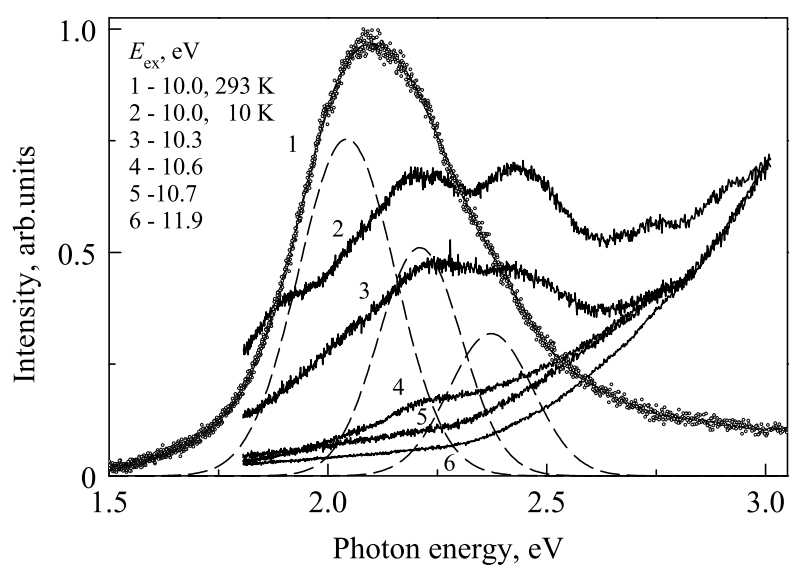

Figure 7. PL spectra of the $\mathrm{SrMgF}_{4}$ crystals measured at temperatures $T=$ (1) 293 and (2-6) $10 \mathrm{~K}$ for different excitation energies $E_{\text {ex }}$. Curves 2-6 are normalized arbitrarily for better viewing. Dashed

lines show the main elementary components obtained by decomposition of spectrum 1 .

by the expression

$$
\delta \hat{\varepsilon}_{j}=\frac{M_{j}}{E_{j}^{2}-E^{2}-i \Gamma_{j} E},
$$

where $i$ is the imaginary unit; $E$ is the excitation energy; $E_{j}, \Gamma_{j}$, and $M_{j}$ are the spectral parameters of the oscillator: the position of the maximum, FWHM, and the amplitude, respectively. The total contribution of electronic transitions outside of the measured spectrum is taken into account by two additional oscillators with indices $j=r$ (red) for the low-energy region and $j=b$ (blue) for the high-energy region:

$$
\begin{aligned}
\hat{\varepsilon}(E)= & \varepsilon_{\infty}+\frac{M_{r}}{E_{r}^{2}-E^{2}-i \Gamma_{r} E}+ \\
& +\frac{M_{b}}{E_{b}^{2}-E^{2}-i \Gamma_{b} E}+ \\
& +\sum_{j} \frac{M_{j}}{E_{j}^{2}-E^{2}-i \Gamma_{j} E},
\end{aligned}
$$

where the summation over $j=1.7$ takes into account the contribution of seven electronic transitions revealed in our measurements; $\varepsilon_{\infty}$ is the high-frequency dielectric constant.

The complex refractive index is related to the dielectric function by the equality $\hat{\varepsilon}=\hat{n}^{2}$. Here, $\hat{n}=n+i k$, where $n$ and $k$ are the refractive and absorption indices, respectively. For a given angle of incidence $\theta$, the reflectivity of $s$-polarized light in a vacuum can be calculated according to the formula [18]

$$
R(E)=\left|\frac{\cos \theta-\hat{n} \cos \hat{\theta}}{\cos \theta+\hat{n} \cos \hat{\theta}}\right|^{2} .
$$




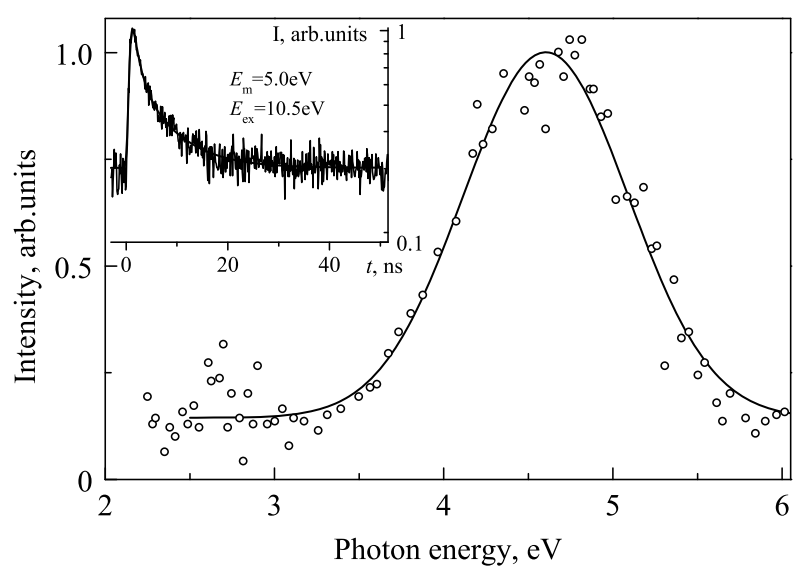

Figure 8. Spectrum of the fast component of the PL decay kinetics for sample SMF\#1 at $T=10 \mathrm{~K}$ and $E_{\text {ex }}=10.5 \mathrm{eV}$. The inset shows an example of the PL decay kinetics recorded for the point at $E_{\mathrm{m}}=5.0 \mathrm{eV}$.

The complex refraction angle is given by the Snell's law $\sin \theta=\hat{n} \sin \hat{\theta}$.

The quality of the approximation of the experimental results was evaluated by the formula

$$
D=\frac{1}{N} \sum_{l=1}^{N} \frac{\left|R_{m l}-R_{c l}\right|}{R_{m l}}
$$

where $R_{m l}$ and $R_{c l}$ are the measured (m) and calculated (c) reflectivity spectra, respectively, and $N$ is the number of experimental points. The results of the best fit, which are presented in Table 2 and Fig. 2, correspond to $D=0.015(10 \mathrm{~K})$ and $0.006(293 \mathrm{~K})$. Below, we will discuss the possible interpretation of the electronic transitions of the oscillators $j=1-7$.

As follows from Table 2, the characteristic 'excitonic' difference between the extrema at 11.26$11.76 \mathrm{eV}(10 \mathrm{~K})$ and $11.10-11.64 \mathrm{eV}(293 \mathrm{~K})$ in the reflectivity spectrum is due to the electronic transitions of the oscillators $E_{2}$ and $E_{3}$. When cooling in the temperature range from 293 to $10 \mathrm{~K}$, the amplitudes of these oscillators increase by factors of 1.7 and 3.1, respectively, and the average temperature coefficients of the shifts of the energy positions of these transitions are $\partial E_{k} / \partial T \mathrm{~T}=-1.3 \times 10^{-4}$ and $-0.2 \times 10^{-4} \mathrm{eV} / \mathrm{K}$, respectively. The order of magnitude of the temperature coefficients is typical of large-radius excitons in wideband-gap crystals [19]. This suggests that the two electronic transitions $E_{2}$ and $E_{3}$ in the SMF crystal are comparable in energy to the excitation of unrelaxed excitons whose energies at $10 \mathrm{~K}$ are $E_{n=1}=11.24$ and $11.37 \mathrm{eV}$, respectively.

The $\mathrm{SrMgF}_{4}$ crystals belong to the $\mathrm{SrF}_{2}-\mathrm{MgF}_{2}$ system. The binary components of this system crystallize in the cubic fluorite $\left(\mathrm{SrF}_{2}\right)$ and tetragonal rutile $\left(\mathrm{MgF}_{2}\right)$ structures [2]. Although the crystallographic structure of the SMF crystals formally differs from the

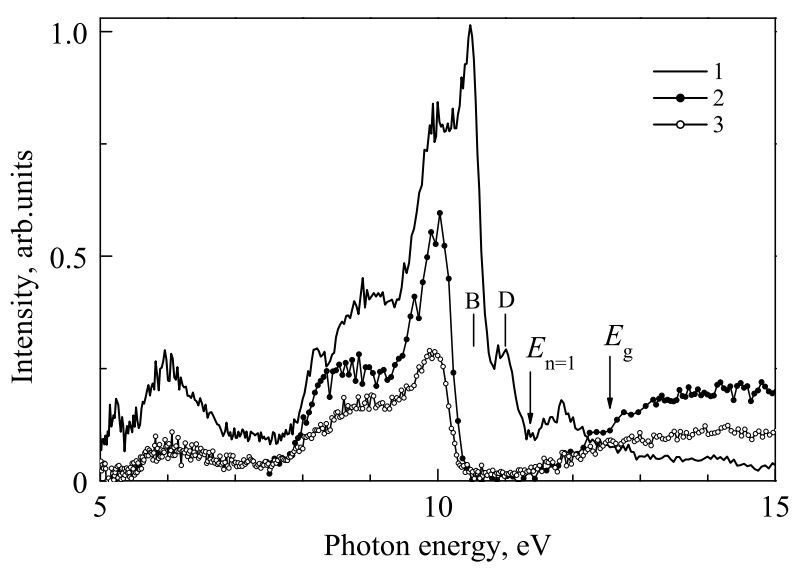

Figure 9. PL excitation spectra of the $\mathrm{SrMgF}_{4}$ crystal (sample SMF\#1) measured at $T=$ (1) 10 and $(2,3) 293 \mathrm{~K}$ and $E_{\mathrm{m}}=$ (1) 2.2 , (2) 2.1 , and (3) $2.5 \mathrm{eV}$. Letters B and D designate the selected PL excitation bands. Vertical arrows indicate the band gap $E_{\mathrm{g}}$ and the position of the first exciton peak $E_{\mathrm{n}=1}$.

structures of $\mathrm{SrF}_{2}$ and $\mathrm{MgF}_{2}$, the electronic structure of SMF should inherit some of the specific features of the electronic structures of the binary components. In terms of the SMF crystal structure, the main translational motif in SMF is a continuous network of $\mathrm{MgF}_{6}$ octahedra. This suggests that a decisive contribution to the SMF electronic structure in this energy range is made by the $\mathrm{MgF}_{2}$ binary component. In the absence of calculated data, we discuss qualitatively the hypothetical contribution of the binary components. Table 3 presents the available data on binary crystals in comparison with our experimental data on the SMF

Table 2. Parameters of the approximation of reflectivity spectra in the region of the fundamental absorption edge of the $\mathrm{SrMgF}_{4}$ crystal

\begin{tabular}{ccccccc}
\hline Oscil- & \multicolumn{3}{c}{$293 \mathrm{~K}$} & \multicolumn{3}{c}{$10 \mathrm{~K}$} \\
\cline { 2 - 7 } lator & $E_{j}$ & \multicolumn{1}{c}{$M_{j}$} & $\Gamma_{j}$ & $E_{j}$ & $M_{j}$ & $\Gamma_{j}$ \\
\hline 1 & 11.05 & 7.536 & 0.46 & 11.00 & 7.838 & 0.60 \\
2 & 11.20 & 2.993 & 0.34 & 11.24 & 5.001 & 0.34 \\
3 & 11.37 & 0.608 & 0.27 & 11.37 & 1.867 & 0.27 \\
4 & 12.12 & 2.207 & 0.56 & 12.18 & 2.252 & 0.41 \\
5 & 12.49 & 5.361 & 0.78 & 12.55 & 7.575 & 0.74 \\
6 & 12.95 & 12.720 & 1.07 & 13.10 & 15.000 & 1.07 \\
7 & 13.50 & 13.620 & 1.17 & 13.79 & 13.890 & 1.26 \\
\hline$r$ & 10.79 & 7.219 & 0.79 & 10.53 & 7.197 & 0.87 \\
$b$ & 14.02 & 6.104 & 0.85 & 14.07 & 0.223 & 0.20 \\
\hline$\varepsilon_{\infty}$ & \multicolumn{4}{c}{1.696} & & 1.638 \\
\hline
\end{tabular}

The parameters of the spectral line of the transition: $E_{j}$ is the position of the maximum, $\mathrm{eV} ; \Gamma_{j}$ is the full width at half-maximum (FWHM), eV; $M_{j}$ is the amplitude; and $j$ is the serial number of the transition. 
Table 3. Electronic structure parameters $(\mathrm{eV})$ of the $\mathrm{SrF}_{2}, \mathrm{MgF}_{2}$, and $\mathrm{SrMgF}_{4}$ crystals at $10 \mathrm{~K}$.

\begin{tabular}{lccc}
\hline \multirow{2}{*}{ Parameter } & \multicolumn{3}{c}{ Crystal } \\
\cline { 2 - 4 }$E_{\mathrm{g}}, \mathrm{eV}$ & $\mathrm{SrF}_{2}$ & $\mathrm{MgF}_{2}$ & $\mathrm{SrMgF}_{4}$ \\
& $11.44^{c}$ & $13.4(\mathbf{E} \perp \mathbf{C})^{b}$ & 12.55 \\
& $11.25^{a}$ & $12.8(\mathbf{E} \| \mathbf{C})^{b}$ & 12.18 \\
\hline$E_{n=1}, \mathrm{eV}$ & & $12.1(\mathbf{E} \perp \mathbf{C})^{b}$ & 11.37 \\
& $10.6^{c}$ & $11.6(\mathbf{E} \| \mathbf{C})^{b}$ & 11.24 \\
\hline$E_{\mathrm{ex}}, \mathrm{eV}$ & $10.4^{d}$ & $11.75^{e}$ & $11.0(\mathrm{D})$ \\
$($ Exciton$)$ & & $11.25^{e}$ & $10.7(\mathrm{C})$ \\
\hline$E_{\mathrm{ex}}, \mathrm{eV}$ & & & $10.5(\mathrm{~B})$ \\
$(\mathrm{CT})$ & & & $10.2(\mathrm{~A})$ \\
\hline$E_{\mathrm{m}}, \mathrm{eV}(\tau)$ & $3.5(8.2 \mathrm{~ns})^{d}$ & $8.4(1.7 \mathrm{~ns})^{e}$ & $4.6(1.5 ; 7.0 \mathrm{~ns})$ \\
& $4.1(59 \mu \mathrm{s})^{c}$ & $3.2(6.4 \mathrm{~ms})^{f}$ & $3.1 ; 3.6 ; 5.0(\mathrm{slow})$ \\
\hline
\end{tabular}

$E_{\mathrm{g}}$ is the minimum energy of interband transitions; $E_{n=1}$ is the position of the maximum of the first exciton absorption peak; $E_{\text {ex }}$ is the position of the maximum of the PL excitation band; $E_{\mathrm{m}}$ is the position of the maximum of the intrinsic PL band; and $\tau$ is the PL decay time. Letters A-D correspond to the notation in Fig. 6; CT is the band of charge-transfer transitions. References to the literature data: ${ }^{a}[21] ;{ }^{b}[34] ;{ }^{c}[35] ;{ }^{d}[36] ;{ }^{e}[37] ;{ }^{f}[38]$.

crystal. The energy positions of the first exciton peak of the $\mathrm{MgF}_{2}$ crystal for transitions with polarizations $\mathbf{E} \| \mathbf{C}$ and $\mathbf{E} \perp \mathbf{C}$ (Table 3) are comparable to the energies of the electronic transitions $E_{2}$ and $E_{3}$ in the SMF crystal. By assuming that the exciton binding energies in $\mathrm{MgF}_{2}$ and $\mathrm{SMF}$ crystals are close to each other, we can compare the energies of the electronic transitions $E_{4}$ and $E_{5}$ with the values of $E_{\mathrm{g}}$ for $\mathbf{E} \| \mathbf{C}$ and $\mathbf{E} \perp \mathbf{C}$, respectively (Table 3 ). It should be noted that, in our measurements, the samples were mounted without controlling the orientation of their crystallographic axes with respect to the polarization vector of the exciting radiation; hence, the spectra can exhibit excitation of the electronic transitions with different polarizations. In this case, the electronic transitions $E_{6}$ and $E_{7}$ with energies higher than $E_{\mathrm{g}}$ should be attributed to interband transitions in the SMF crystal.

The spectra of the optical constants calculated by the Kramers-Krönig method are shown in Fig. 3. We are unaware of experimental data on refractive indices of SMF crystals. Therefore, we used the available data on related crystals. For $E=7.87 \mathrm{eV}$, the refractive indices are equal to $1.6138\left(\mathrm{BaMgF}_{4}[26]\right), 1.621\left(\mathrm{SrF}_{2}\right.$ [27]), $1.4464\left(\mathrm{MgF}_{2}\right.$ [27]), and 1.632 for $\mathrm{SrMgF}_{4}$ in our calculations. The minimum threshold energies, at which $n(E)=1$, are equal to $13.5\left(\mathrm{SrF}_{2}\right.$ [27]), 22.2 $\left(\mathrm{MgF}_{2}\right.$ [27]), and $19.9 \mathrm{eV}$ for SMF in our calculations. Furthermore, the calculated spectrum of the refractive index $\mathrm{n}(\mathrm{E})$ for the SMF crystal is in relatively good agreement with the profiles of the available spectra of the refractive index for $\mathrm{MgF}_{2}$ in this energy range
[27]. In the $k(E)$ spectrum, the lowest energy peak at $11.18 \mathrm{eV}$ has an excitonic nature. It is a superposition of the electronic transitions $E_{2}$ and $E_{3}$. In the energy range from 12.2 to $12.7 \mathrm{eV}$, the observed increase in the intensity of the spectra $k(E)$ and $\varepsilon_{2}(E)$ is associated with the onset of the interband transitions $E_{4}$ and $E_{5}$, which we identified with $E_{\mathrm{g}}$ (Table 3 ). With a further increase in the photon energy in the range from $E_{\mathrm{g}}$ to $16 \mathrm{eV}$, the profiles of all the spectra are determined by the specificity of interband transitions in the $\mathrm{SrMgF}_{4}$ crystal. Their reasonable interpretation requires quantum-chemical calculations of the $\mathrm{SMF}$ electronic structure. At this stage, we only note that, in the energy range above $16 \mathrm{eV}$, there is a tendency toward monotonic decrease in the function $\varepsilon_{2}(E)$, which corresponds to the depletion of the sum rule and can indicate the excitation of plasma oscillations in the valence band of the crystal. The electronic transitions in the energy range above $16 \mathrm{eV}$ occur involving the core $\mathrm{Sr} 4 p$ electrons [20]. A comparison of our results with the available data on $\mathrm{SrF}_{2}[20,28]$ allows us to presumably assign the doublet structure at energies of $20-22 \mathrm{eV}$ to the excitation of the cation $\mathrm{Sr} 4 p$ excitons. A broad peak in the reflectivity spectrum at $27-30 \mathrm{eV}$ corresponds to the most intense maxima in the spectra $-\operatorname{Im} \hat{\varepsilon}^{-1}$ and $-\operatorname{Im}(1+\hat{\varepsilon})^{-1}$. It is known [29] that the energy positions of the most intense maxima in these spectra coincide with the energies of the volume $\left(E_{\mathrm{pv}}\right)$ and surface $\left(E_{\mathrm{ps}}\right)$ plasmons. According to our calculations for the SMF crystal, these energies are $E_{\mathrm{pv}}=$ $29.7 \mathrm{eV}$ and $E_{\mathrm{ps}}=28.3 \mathrm{eV}$.

Let us now discuss possible electronic transitions in the lowest energy region of the spectra of the optical constants below the energy of the first exciton peak $E_{n=1}$. From Table 3 it follows that the contribution of the $\mathrm{SrF}_{2}$ binary component should be expected at energies that are approximately $1 \mathrm{eV}$ lower than those for $\mathrm{MgF}_{2}$. In the SMF electronic structure, these energy levels are responsible for the $\mathrm{F}-\mathrm{Sr}$ charge-transfer transitions. Similar properties are exhibited, for example, by the electronic structure of the $\beta-\mathrm{BaBO}_{4}$ crystal. For this crystal, the exciton peak, which corresponds to the lowest energy electronic excitations of the boron-oxygen anionic group, is observed at $7.5 \mathrm{eV}$ [30], whereas the low-energy part of the fundamental absorption tail of the $\beta-\mathrm{BaBO}_{4}$ crystal in the energy range of $6.2-6.8 \mathrm{eV}$ is associated with the $\mathrm{O}-\mathrm{Ba}$ charge-transfer transitions of lower intensity [31]. In the SMF crystal, there are six nonequivalent positions for Sr atoms, which differ by their coordination numbers and bond lengths. Owing to the influence of the disordering in the $\mathrm{SrF}_{2}$ component, we should expect the existence of several different $\mathrm{F}$-Sr charge-transfer transitions, which differ in energy and occur below the energy of the exciton peak. These properties are inherent in the electronic transition $E_{1}$, which is essentially necessary for the correct fitting of the experimental spectra but cannot be assigned to the excitonic tran- 


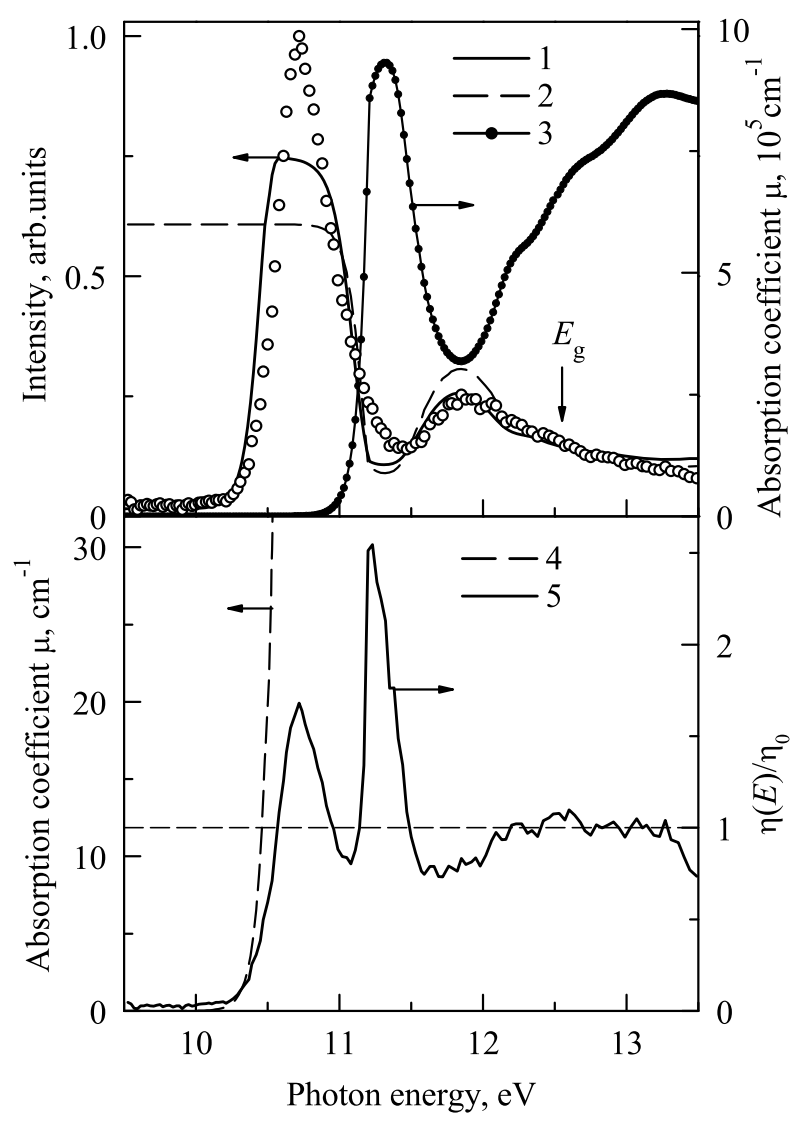

Figure 10. PL excitation spectra of the $\mathrm{SrMgF}_{4}$ crystal (sample SMF\#1). Open circles represent the spectrum measured at $T=10 \mathrm{~K}$ and $E_{\mathrm{m}}=3.64 \mathrm{eV}$.

Curve 1 is the spectrum calculated in the framework of the model representations $(L=70 \mathrm{~nm}$ and $d=$ $1 \mathrm{~mm})$. Also shown for comparison are the correction function $y_{0}(E)(\Delta=20 \mathrm{~nm})$ (curve 2), the calculated optical absorption spectrum $\mu(E)$ (curve 3), the enlarged image of this spectrum in the fundamental absorption edge region $0-30 \mathrm{~cm}^{-1}$ (curve 4), and the relative PL quantum yield $\eta(E) / \eta_{0}$ (curve 5 ). The vertical arrow indicates the band gap $E_{\mathrm{g}}$.

sitions according to the following features: the amplitude is almost independent of the temperature, the coefficient of the temperature shift has the opposite sign, and the bandwidth of the oscillator significantly increases during cooling to $10 \mathrm{~K}$ (Table 2). On this basis, the electronic transitions $E_{1}$ and, possibly, $\mathrm{Er}$ should be attributed to the $\mathrm{F}-\mathrm{Sr}$ charge-transfer transitions.

\subsection{Optical Transitions and Excitation of the Luminescence}

In the above context, we can reasonably interpret selective bands in the PL excitation spectra at the low- energy tail of the fundamental absorption of the crystal. A comparison of the spectra of the optical constants for SMF (Fig. 3) and for $\mathrm{SrF}_{2}$ and $\mathrm{MgF}_{2}$ [27] indicates that the numerical values of the parameters of the SMF electronic structure lie in the range between the corresponding values for $\mathrm{SrF}_{2}$ and $\mathrm{MgF}_{2}$. We are unaware of data on the optical absorption spectra at the low-energy tail of the fundamental absorption of the SMF crystal. However, the available data on the related crystals give the following values: $8.8 \mathrm{eV}$ for $\mathrm{BaMgF}_{4}$ [26], $9.5 \mathrm{eV}$ for $\mathrm{SrF}_{2}$ [32], and $11.0 \mathrm{eV}$ for $\mathrm{MgF}_{2}$ [33]. Therefore, the energy range from 10.0 to $10.5 \mathrm{eV}$ is a reasonable estimate for the low-energy edge of the fundamental absorption of the SMF crystal.

We now discuss the interpretation of the dominant $\mathrm{C}$ band in the PL excitation spectra (Fig. 6). It is well known that many properties of PL excitation spectra in the vacuum ultraviolet spectral region are determined by the so-called surface energy losses. In terms of simple diffusion concepts [25,34], the profile of the PL excitation spectrum can be described by the expression

$$
I(E)=\eta(E)(1-R(E)) \frac{1-\exp (-\mu(E) d)}{1+\mu(E) L},
$$

where $\eta(E)$ is the PL quantum yield in the bulk of the crystal, $R$ is the reflection coefficient, $\mu$ is the absorption coefficient, $L$ is the diffusion length of electronic excitations, and $d$ is the thickness of the crystal. We calculated the absorption spectrum $\mu(E)$ from the spectra of the optical constants (Fig. 3). A comparison of the measured PL excitation spectrum with the results of the calculation using formula (5) (Fig. 10) allows us to draw the following conclusions. First, in the SMF crystal, there are mobile electronic excitations, i.e., excitons, the average diffusion length of which according to our calculations reaches $70 \mathrm{~nm}$. The profile of the band at $10.7 \mathrm{eV}$ in the PL excitation spectrum (Fig. 3) is determined by two competing processes. The low-energy slope of this band is determined by the factor $(1-\exp (-\mu(E) d))$ and corresponds to an increase of the optical absorption up to several tens of inverse centimeters at the low-energy tail of the fundamental absorption of the crystal. The high-energy slope of this band and the PL excitation spectrum at higher energies are determined by the denominator $(1+\mu(E) L)$, which describes the surface energy losses. Owing to this factor, the PL excitation spectrum can be modulated by the function $\mu(E)$. The modulation effect explains, in particular, the presence of local extrema at energies of 11.45 and $11.85 \mathrm{eV}$ and the subsequent gradual decline in the PL excitation spectrum (Fig. 10). Second, the calculations performed at $\eta(E)=$ const do not explain all the observed features of the PL excitation spectrum. For the correct description of the PL excitation spectra, it is necessary to take into account the energy dependence of 
the PL quantum yield. From the experimental data, we can estimate the dependence $\eta(E) / \eta_{0}$, which is obtained by dividing the PL excitation spectrum by the correction function $y_{0}(E)[36]$ :

$$
y_{0}(E)=A \exp (-\mu(E) \Delta),
$$

where $A$ is the scaling factor and $\Delta$ is the thickness of the 'dead' layer, which determines the surface energy losses. These parameters can be obtained through the best fit of the experimental spectrum by the function $y_{0}(E)$ in the energy range $E>E_{\mathrm{g}}$, where the PL quantum yield can be considered to be constant $3 \eta(E)=\eta_{0}$. The function $\eta(E) / \eta_{0}$ obtained for $\Delta=$ $20 \mathrm{~nm}$ is shown in Fig. 10. It can be seen from Fig. 10 that the function $\eta(E) / \eta_{0}$ increases rapidly from almost zero at the fundamental absorption edge and tends to unity at energies $\mathrm{E}>11.5 \mathrm{eV}$. As is also seen, there are two intense peaks at 10.7 and $11.2 \mathrm{eV}$, whose energy positions do not coincide with the energies of the oscillators (Table 2). In our opinion, the origin of these peaks cannot be explained by the contribution to the PL quantum yield $\eta(E)$ from the direct photoexcitation of electronic transitions corresponding these oscillators.

In contrast, the energy positions of the B and D bands in the PL excitation spectra (Fig. 6) coincide with the energies of the oscillators $E_{r}$ and $E_{1}$ (Table 2), their origin cannot be explained by surface energy losses, and the profiles of the bands cannot be approximated by formula (5) for reasonable values of the fitting parameters. The $\mathrm{B}$ and $\mathrm{D}$ bands partially overlap with the dominant $\mathrm{C}$ band. As can be seen from Fig. 6, the high-energy slope of the B band is antisymmetric with respect to the low-energy slope of the $\mathrm{C}$ band; i.e., an increase in the intensity of one band occurs with a decrease in the intensity of the other band. This can indirectly indicate a competition between the relaxation processes responsible for the $\mathrm{B}$ and $\mathrm{C}$ bands. In our opinion, the bands at 10.5 and $11.0 \mathrm{eV}$ in the PL excitation spectrum are associated with the direct photoexcitation of the oscillators $E_{r}$ and $E_{1}$ (Table 2) in the SMF crystal. The maxima of these lines in the PL excitation spectrum are located below the energy positions of the exciton absorption peaks $E_{2}=11.24 \mathrm{eV}$ and $E_{3}=11.37 \mathrm{eV}$ (Table 3). The band at $11.0 \mathrm{eV}$ always has a relatively low intensity or manifests itself in the form of a 'shoulder' on the highenergy slope of the dominant band at $10.7 \mathrm{eV}$. This is explained by the fact that the excitation band at $11.0 \mathrm{eV}$ is located not at the fundamental absorption edge of the crystal, where the absorption coefficient is relatively small $\left(30-50 \mathrm{~cm}^{-1}\right)$, but it lies in the energy region far enough from the edge, where the absorption coefficient is significantly higher and, accordingly, the mean free path of photons in the crystal is shorter, which leads to an increase in the nonradiative energy losses of electronic excitations on the surface of the crystal.
The relatively high absorption coefficient in the region of the excitation band at $11.0 \mathrm{eV}$ is determined by two factors. First, in the vicinity of the excitation band, there are two exciton absorption bands at 11.24 and $11.47 \mathrm{eV}$. Second, from Table 3 it follows that the energy range of 10.6-11.2 eV corresponds to the excitation threshold for low-energy electronic transitions in the $\mathrm{SrF}_{2}$ binary component. Therefore, in this region, we can expect $\mathrm{F}$-Sr charge-transfer transitions. In our opinion, the excitation band at $10.5 \mathrm{eV}$ in the SMF crystal (Table 3 ) can be tentatively attributed to the F-Sr charge-transfer transitions in the SMF lattice disordered for strontium. Possibly, the same is also true for the A band at $10.2 \mathrm{eV}$. All the PL excitation bands located at lower energies should be attributed to optical transitions in unidentified lattice defects (color centers based on anion vacancies, structural defects of cation sublattices, and impurity defects).

\subsection{Luminescence Spectra}

All the observed low-temperature luminescence bands can be classified into two groups according to their PL excitation spectra in the vicinity of the dominant $\mathrm{C}$ band.

The first group includes luminescence bands at 2.6$3.3 \mathrm{eV}$ (II) and 3.3-4.2 eV (III), with the excitonic C band dominating in their excitation spectra (Fig. 6). These PL bands are potential candidates for further analysis of their possible intrinsic nature. Note, however, that the same properties can be observed in the PL bands of defects, for which the main excitation channel is the energy transfer of electronic excitations through the exciton migration. Complex multicomponent crystals, where there are charge-transfer transitions between different fragments of the crystal structure, often exhibit an efficient excitation of intrinsic PL bands in the region of charge-transfer transitions, as well as the complete or partial suppression of the excitation of these bands in the excitonic region. For example, the intrinsic PL excitation spectrum of the $\mathrm{Li}_{6} \mathrm{GdB}_{3} \mathrm{O}_{9}$ crystal is dominated by the band of $\mathrm{O}-\mathrm{Gd}$ charge-transfer transitions at $6.8 \mathrm{eV}$, whereas the excitation band of the free exciton in the range of $8-9 \mathrm{eV}$ appears only in the reflectivity spectrum and is almost completely suppressed in the intrinsic PL excitation spectrum [37-39]. Therefore, in the interpretation of bands in the PL spectra of the SMF crystal, it should be remembered that the intrinsic PL bands of SMF can also be excited in the energy region of the charge-transfer transitions.

The second group includes luminescence bands at 1.8-2.6 eV (I) and 4.2-5.5eV (IV). The excitation spectra of these bands are antibate with respect to the excitonic $\mathrm{C}$ band (Figs. 6, 9). The excitation of these PL bands occurs in the transparency region of the crystal, 
as well as upon direct photoexcitation of the transitions corresponding to the oscillators $E_{r}(10.5 \mathrm{eV})$ and $E_{1}(11.0 \mathrm{eV})$. This indicates their relation with lattice defects of the SMF crystal. The nature of radiative transitions in defects of SMF crystals requires a separate detailed investigation.

\section{CONCLUSIONS}

The optical-quality $\mathrm{SrMgF}_{4}$ single crystals were grown by the Bridgman method, and their crystal structure was analyzed. The electronic structure of the $\mathrm{SrMgF}_{4}$ crystals and the radiative relaxation of electronic excitations were investigated using lowtemperature $(T=10 \mathrm{~K})$ time-resolved fluorescence optical and vacuum ultraviolet spectroscopy under excitation by synchrotron radiation in the energy range of $3.7-36.0 \mathrm{eV}$. This optical material exhibits an intense luminescence over a wide (from red to ultraviolet) region of the spectrum, which is efficiently excited in the region of the low-energy fundamental absorption edge. Based on the low-temperature reflectivity spectra and calculations of the optical constants, the following parameters of the electronic structure were determined for the first time: the minimum energy of interband transitions $E_{\mathrm{g}}=12.55 \mathrm{eV}$, the position of the first exciton peak $E_{n=1}=11.37 \mathrm{eV}$, the position of the maximum of the 'exciton' luminescence excitation band at $10.7 \mathrm{eV}$, and the position of the fundamental absorption edge at $10.3 \mathrm{eV}$. The $\mathrm{SrMgF}_{4}$ lattice predominantly disordered over the strontium atoms is characterized by the presence of unidentified defects, which actively capture electronic excitations. Upon excitation above $E_{\mathrm{g}}$, there is no efficient energy transfer from the matrix to luminescence centers. The radiative relaxation of excited states of the defects is responsible for the luminescence bands in the ranges of $1.8-2.6$ (I) and $4.2-5.5 \mathrm{eV}$ (IV). The main excitation channel of the luminescence bands at 2.6-3.3 (II) and $3.3-4.2 \mathrm{eV}$ (III) is the energy transfer of electronic excitations through the exciton migration. Therefore, these luminescence bands can be considered as potential candidates for further analysis of their possible intrinsic nature.

\section{ACKNOWLEDGMENTS}

We would like to thank D.Yu. Naumov (Nikolaev Institute of Inorganic Chemistry, Siberian Branch of the Russian Academy of Sciences, Novosibirsk, Russia) for his assistance in performing the structural investigations.

This study was supported in part by the Ministry of Education and Science of the Russian Federation (grant no.14.A18.21.0076), the Siberian Branch of the Russian Academy of Sciences (grant no. 28), the Hamburg Synchrotron Radiation Laboratory HASYLAB at DESY (Hamburg, Germany) (project no. 20110843), and the European Social Fund (Mobilitas grant no. MJD219).

\section{REFERENCES}

1. E. Banks, S. Nakajima, and M. Shone, J. Electrochem. Soc. 127, 2234 (1980).

2. Q. Bingyi and E. Banks, Mater. Res. Bull. 17, 1185 (1982).

3. N. Ishizawa, K. Suda, B. E. Etschmann, T. Oya, and N. Kodama, Acta Crystallogr., Sect. C: Cryst. Struct. Commun. 57, 784 (2001).

4. S. C. Abrahams, Acta Crystallogr., Sect. B: Struct. Sci. 58, 34 (2002)

5. M. Yamaga, K. Itoh, S. Yabashi, Y. Masui, S. Ono, M. Sakai, and N. Sarukura, UVSOR Act. Rep. 2003, 57 (2004).

6. M. Yamaga, E. Hayashi, N. Kodama, K. Itoh, S. Yabashi, Y. Masui, S. Ono, N. Sarukura, T. P. J. Han, and H. G. Gallagher, J. Phys.: Condens. Matter 18, 6033 (2006).

7. H. Hagemann, F. Kubel, H. Bill, and F. Gingl, J. Alloys Compd. 374, 194 (2004).

8. F. Kubel, H.-R. Hagemann, and H. Bill, Mater. Res. Bull. 32, 263 (1997).

9. Y. Wu and C.-S. Shi, Solid State Commun. 95, 319 (1995).

10. Y. Wu and C.-S. Shi, Acta Phys.-Chim. Sin. 11, 907 (1995).

11. C. Veitsch, F. Kubel, and H. Hagemann, Mater. Res. Bull. 43, 168 (2008).

12. P. Muller, R. Herbst-Irmer, A. L. Spek, T. R. Schneider, and M. R. Sawaya, Crystal Structure Refinement: A Crystallographer's Guide to SHELXL (Oxford University Press, New York, 2006).

13. N. E. Kashcheeva, D. Y. Naumov, and E. V. Boldyreva, Z. Kristallogr. 214, 534 (1999).

14. G. Zimmerer, Radiat. Meas. 42, 859 (2007).

15. V. Lucarini, J. J. Saarinen, K. E. Peiponen, and E. M. Vartiainen, Kramers-Kronog Relations in Optical Materials Research (Springer-Verlag, Berlin, 2005).

16. M. Weissbluth, Atoms and Molecules (Academic, New York, 1978).

17. P. W. Milonni and J. H. Eberly, Laser Physics (Wiley, Hoboken, New Jersey, United States, 2010).

18. M. Born and E. Wolf, Principles of Optics (Pergamon, New York, 1980).

19. E. F. Gross, Investigations on the Optics and Spectroscopy of Crystals and Liquids: Selected Works (Nauka, Leningrad, 1976) [in Russian].

20. G. W. Rubloff, Phys. Rev. B: Solid State 5, 662 (1972).

21. C. Jouanin, J. P. Albert, and C. Gout, J. Phys. (Paris) 37, 595 (1976).

22. A. K. S. Song and R. T. Williams, Self-Trapped Excitons (Springer-Verlag, Berlin, 1996).

23. K. V. Ivanovskikh, V. A. Pustovarov, and B. V. Shulgin, Nucl. Instrum. Methods Phys. Res., Sect. 
A 543, 229 (2005).

24. V. N. Kolobanov, V. V. Mikhailin, S. P. Chernov, D. A. Spassky, V. N. Makhov, M. Kirm, E. Feldbach, and S. Vielhauer, J. Phys.: Condens. Matter 21 (6), 375501 (2009)

25. L. F. Chen, L. Zhou, and K. S. Song, J. Phys.: Condens. Matter. 9, 6633 (1997).

26. S. C. Buchter, T. Y. Fan, V. Liberman, J. J. Zayhowski, M. Rothschild, E. J. Mason, A. Cassanho, H. P. Jenssen, and J. H. Burnett, Opt. Lett. 26, 1693 (2001).

27. H. H. Li, J. Phys. Chem. Ref. Data 9, 161 (1980).

28. G. W. Rubloff, J. Freeouf, H. Fritzsche, and K. Murase, Phys. Rev. Lett. 27, 361 (1971).

29. D. Pines, Elementary Excitations in Solids : Lectures on Phonons, Electrons, and Plasmons (W. A. Benjamin, New York, 1963; Mir, Moscow, 1963).

30. V. Kisand, R. Kink, M. Kink, J. Maksimov, M. Kirm, and I. Martinson, Phys. Scr. 54, 542 (1996).
31. W.-D. Cheng, J.-S. Huang, and J.-X. Lu, Phys. Rev. B: Condens. Matter 57, 1527 (1998).

32. T. Tomiki and T. Miyata, J. Phys. Soc. Jpn. 27, 658 (1969).

33. M. W. Williams, R. A. MacRae, and E. T. Arakawa, J. Appl. Phys. 38, 1701 (1967).

34. A. Belsky and J. C. Krupa, Displays 19, 185 (1999).

35. A. N. Vasil'ev and V. V. Mikhailin, Introduction to Spectroscopy of Dielectrics (Yanus-K, Moscow, 2000) [in Russian].

36. T. Matsumoto, M. Shirai, and K. Kan'no, J. Phys. Soc. Jpn. 64, 987 (1995).

37. I. N. Ogorodnikov and V. A. Pustovarov, J. Lumin. 134, 113 (2013).

38. I. N. Ogorodnikov and V. A. Pustovarov, J. Phys.: Condens. Matter. 24 (8), 405902 (2012).

39. I. N. Ogorodnikov and V. A. Pustovarov, JETP Lett. 96 (5), 308 (2012). 